C6

mice fed a soft diet showed a significant reduction specifically of gingival $T_{H} 17$ cells 5
T CELLS

\section{Something to chew on}

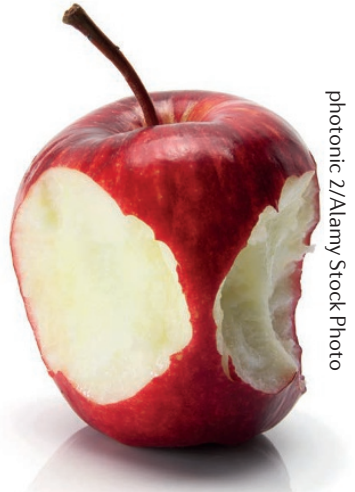

Immune cell populations at barrier surfaces such as the skin and gut are regulated by local cues that ensure microbial defence and homeostasis. Yet little is known about the cues regulating immune cells at a key barrier surface in the mouth - the gingiva. A new study shows that mechanical damage from chewing (mastication) promotes an accumulation of Thelper $17\left(\mathrm{~T}_{H} 17\right)$ cells with age. These cells seem to support host protection but when amplified can also contribute to periodontal bone loss.

In healthy individuals, $\mathrm{T}_{\mathrm{H}} 17$ cells are enriched in gut and skin barrier tissues and are influenced by colonization with commensal microbes. By contrast, few $\mathrm{T}_{\mathrm{H}} 17$ cells were seen in the gingiva of young (8-week-old) mice. However, by 24 weeks of age (equivalent to middle age), $T_{H} 17$ cell frequencies and total numbers were significantly increased. This age-dependent expansion of gingival $T_{H} 17$ cell populations was independent of oral microbes, because the increase also occurred in germ-free mice and in mice lacking segmented filamentous bacteria (known inducers of $\mathrm{T}_{\mathrm{H}} 17$ cells in the gut). Also unlike gut or skin $T_{H} 17$ cell development, the authors found no role for interleukin-1 (IL-1) or IL-23 in the development of gingival $T_{H} 17$ cells. Instead, IL-6 was required for gingival $T_{H} 17$ cell accumulation, as mice with $T$ cells lacking IL-6 receptor did not generate $T_{H} 17$ cells in the gingiva.

The authors then investigated whether mechanical damage resulting from mastication could be involved in gingival $\mathrm{T}_{\mathrm{H}} 17$ cell generation by feeding weanling mice a nutritionally matched soft diet. At 24 weeks of age, mice fed a soft diet showed a significant reduction specifically of gingival $T_{H} 17$ cells compared with mice fed normal chow. Conversely, mice subjected to increased mechanical damage of the gingiva, through rubbing with a cotton applicator every other day or by placing them on a hardened diet, had more gingival $\mathrm{T}_{H} 17$ cells than age-matched control mice. The damage-induced increase in $T_{H} 17$ cells was associated with rapid production of IL- 6 by epithelial cells in the gingiva.

Finally, the authors showed that physiological damage to the gingiva through mastication and the accumulation of $\mathrm{T}_{\mathrm{H}} 17$ cells was sufficient to drive the expression of epithelial defensins and neutrophil chemoattractants implicated in host defence. However, a pathogenic consequence for elevated $T_{H} 17$ cells was suggested by the increase in periodontal bone loss observed in 24- compared with 8-week-old wild-type mice but not in 24-week-old IL-17-deficient mice. Together, these data identify physiological and pathological roles for mastication-induced gingival $\mathrm{T}_{\mathrm{H}} 17$ cells.

LucyBird 